\title{
A distinguishing gravitational property for gravitational equation in higher dimensions
}

\author{
Naresh Dadhich ${ }^{1,2, a}$ \\ ${ }^{1}$ Centre for Theoretical Physics, Jamia Millia Islamia, New Delhi 110025, India \\ ${ }^{2}$ Inter-University Centre for Astronomy and Astrophysics, Post Bag 4, Pune 411 007, India
}

Received: 19 October 2015 / Accepted: 4 February 2016 / Published online: 26 February 2016

(C) The Author(s) 2016. This article is published with open access at Springerlink.com

\begin{abstract}
It is well known that Einstein gravity is kinematic (meaning that there is no non-trivial vacuum solution; i.e. the Riemann tensor vanishes whenever the Ricci tensor does so) in 3 dimension because the Riemann tensor is entirely given in terms of the Ricci tensor. Could this property be universalized for all odd dimensions in a generalized theory? The answer is yes, and this property uniquely singles out pure Lovelock (it has only one $N$ th order term in the action) gravity for which the $N$ th order Lovelock-Riemann tensor is indeed given in terms of the corresponding Ricci tensor for all odd, $d=2 N+1$, dimensions. This feature of gravity is realized only in higher dimensions and it uniquely picks out pure Lovelock gravity from all other generalizations of Einstein gravity. It serves as a good distinguishing and guiding criterion for the gravitational equation in higher dimensions.
\end{abstract}

\section{Introduction}

The absence of all forces is characterized by a maximally symmetric spacetime of constant (homogeneous) curvature, and Einstein gravity (GR) naturally arises when spacetime turns inhomogeneous [1]. It is the Riemann curvature that should determine the dynamics of the force responsible for its inhomogeneity. The Riemann curvature satisfies the Bianchi differential identity, which is purely a differential geometric property and its trace yields a second rank symmetric tensor with vanishing divergence, the Einstein tensor, giving the second order differential operator for the equation of motion. This is how we are uniquely led to the Einstein gravitational equation on identifying the cause of inhomogeneity as a matter-energy distribution, a universal physical property for all that physically exists [1]. Thus gravitational dynamics is entirely determined by curvature and it resides in it.

\footnotetext{
a e-mail: nkd@iucaa.in
}

It is very illuminating that without asking for an equation for gravity, GR simply follows from the geometric properties of Riemann curvature. Similarly, does geometry also determine the spacetime dimension? The second order differential operator in the equation is given by the Einstein tensor, which is non-trivial only in dimensions $d>2$. Next the equation should admit a non-trivial vacuum solution for free propagation, which requires $d>3$. In $d=3$, the Riemann curvature is entirely given in terms of the Ricci tensor; i.e. it vanishes whenever the Ricci tensor vanishes. That is how gravity is kinematic in $d=3$ as the vacuum is flat, and the absence of a non-trivial vacuum solution signifies the absence of free degrees of freedom for propagation of the field. This is how we come to the usual 4 dimensional spacetime that admits a non-trivial vacuum solution. However, the Einstein equation would be valid in all higher dimensions as well.

The kinematic property signifies the fact that there are no free degrees of freedom in 3 dimensions. In 4 dimensions both gravity and electromagnetics have two degrees of freedom, while in 3 dimensions, the former has none but the latter has one. The kinematic property in a specifically odd dimension is therefore unique to gravity. We would like to take it as one of the distinguishing features. We therefore ask the question: could the kinematic property of GR for odd $d=3$ dimensions be universalized to all odd dimensions? Naturally this would require a generalization of GR because GR could be kinematic only in 3 dimensions and not otherwise. However, this gravitational property may serve as a good guiding principle for a gravitational equation in higher dimensions. That is what we shall probe. We shall establish that it uniquely singles out pure Lovelock gravity for which the action consists of only one $N$ th order term of the Lovelock polynomial Lagrangian. Lovelock is the most natural generalization of GR because it is the only one that remarkably retains the second order character of the equation despite the action being a homogeneous polynomial in Riemann. This is quintessentially a higher dimensional generalization of GR. 
In this essay we shall proceed as follows. First, we shall establish that gravity is indeed kinematic [2,3] in all odd, $d=2 N+1$, dimensions in pure Lovelock gravity relative to properly defined Lovelock analogs of the Riemann and Ricci tensors [3-5]. Since this generalization is effective only in higher dimensions, it is therefore pertinent to ask: what is it that requires higher dimension(s), and why is it small so that it is not accessible to present day observations? That is what we shall probe next by appealing to a general principle and some gravitational properties. Next we will argue that the pure Lovelock case is thus a proper gravitational equation [6] in higher dimensions and it is the only one that obeys kinematicity of gravity in all odd, $d=2 N+1$, dimensions. It is valid only for two, odd, $d=2 N+1$, and even, $d=2 N+2$, dimensions. It includes GR in the linear order $N=1$. We shall end with a discussion.

\section{Kinematic property}

Dadhich [4] defined an appropriate Lovelock analog of the Riemann tensor, which was a homogeneous polynomial in the Riemann tensor, with the property that the trace of its Bianchi derivative vanished. That gave rise to a corresponding analog of the Einstein tensor which was the same as the one obtained by varying the corresponding Lovelock action. Using this Lovelock-Riemann generalization it was first shown that static vacuum spacetime was kinematic in all odd, $d=2 N+1$, dimensions; i.e. vanishing of Ricci implied vanishing of Riemann [2]. Right on the heels of this discovery came yet another parallel definition of the Lovelock-Riemann tensor by Kastor [5], which involved a $(2 N, 2 N)$-rank tensor, completely antisymmetric both in its upper and lower indices. Though the two Lovelock-Riemann analogs are not completely equivalent, yet interestingly they both yield the same Einstein tensor, and hence the same equation of motion. The difference between them came to the fore recently while studying vacuum solutions for the Kasner metric [7] where the kinematic property held good for the Kastor Lovelock-Riemann case but not for the Dadhich one. Interestingly, for a static spacetime, the difference between the two vanishes and that is why it was not noticed earlier.

In view of its general validity, we shall employ the KastorLovelock-Riemann tensor for establishing the kinematic property in all odd, $d=2 N+1$, dimensions. This $(2 N, 2 N)$ rank tensor is defined as follows [5]:

$\mathbb{R}_{a_{1} a_{2} \cdots a_{2 N}}^{b_{1} b_{2} \cdots b_{2 N}}=R_{\left[a_{1} a_{2}\right.}^{\left[b_{1} b_{2}\right.} \cdots R_{\left.a_{2 N-1} a_{2 N}\right]}^{\left.b_{2 N-1} b_{2 N}\right]}$

It is a product of $N$ Riemann tensors and completely antisymmetric in both its upper and lower indices. With all indices lowered, it is also symmetric under exchange of both groups of indices, $a_{i} \leftrightarrow b_{i}$.
The Lovelock Lagrangian is written as

$$
\begin{aligned}
\mathcal{L}= & \frac{2^{N}}{(2 N) !(d-2 N) !} \epsilon_{a_{1} a_{2} \cdots a_{d}} \\
& \times \mathbb{R}^{a_{1} a_{2} \cdots a_{2 N}} \wedge e^{a_{2 N+1}} \wedge \cdots \wedge e^{a_{d}},
\end{aligned}
$$

giving rise to the corresponding Einstein tensor

$$
\begin{aligned}
\mathcal{E}_{c}^{b}= & \frac{2^{N}}{(2 N) !(d-2 N-1) !} \epsilon_{a_{1} a_{2} \cdots a_{d-1} c} \\
& \times \mathbb{R}^{a_{1} a_{2} \cdots a_{2 N}} \wedge e^{a_{2 N+1}} \wedge \cdots \wedge e^{a_{d-1}} \wedge e^{b} .
\end{aligned}
$$

It is purely an algebraic property that for $d=2 N+1$, the above defined $4 N$ th order Lovelock-Riemann tensor could be entirely written in terms of its contraction, the Ricci and thereby the Einstein tensor, and it is in fact written as follows [3]:

$\mathbb{R}_{a_{1} \cdots a_{2 N}}^{b_{1} \cdots b_{2 N}}=\frac{1}{(2 N) !} \epsilon^{b_{1} \cdots b_{2 N+1}} \epsilon_{a_{1} \cdots a_{2 N+1}} \mathcal{E}^{a_{2 N+1}} b_{2 N+1}$.

This clearly establishes the kinematic property that the Lovelock-Riemann tensor vanishes in all odd, $d=2 N+$ 1, dimensions whenever the corresponding Einstein (Ricci) tensor vanishes. It may, however, be mentioned that though vacuum spacetime in odd dimensions would be LovelockRiemann flat, it would not in general be Riemann flat [2]. Another way of characterizing the kinematic property is that the corresponding Weyl curvature vanishes in all odd, $d=$ $2 N+1$, dimensions.

\section{Why higher dimensions?}

It is the symmetries of field theory for a consistent theory of fundamental particles and their interactions that lead naturally to higher dimensions, and this paradigm is popularly known as string theory. It is all driven by field theoretic considerations without any direct reference to gravity. Instead I would here like to concern myself only with gravitation to ask the question: are there any gravitational features that have so far remained unaddressed and does their inclusion require higher dimensions?

One such possible feature could be probing of gravity in the high energy regime [1,8-10]. For addressing the high energy effects of any theory, we generally include higher powers of the basic field entity which in the present case is a Riemann tensor. In Einstein gravity, the Riemann tensor occurs linearly in the action, for high energy considerations we should therefore include higher powers of the Riemann tensor in the action. However, at the same time if we demand that the basic character of the equation should not change; i.e. it should continue to remain a second order differential equation which is also required for warding off undesirable features like the occurrence of ghosts. This uniquely sin- 
gles out the Lovelock action, which alone has the remarkable property that the equation continues to remain second order despite the action being a homogeneous polynomial in the Riemann tensor. But higher order Riemann terms in the Lovelock action make a non-zero contribution to the equation only in dimension $>4$. That is why higher dimensions are required to probe the high energy effects of gravity [8-10].

Note that this is a purely a classical argument for higher dimensions.

\section{Gravitational equation in higher dimensions}

Since gravity cannot remain entirely confined to a given dimension, the consideration of higher dimensions becomes pertinent, and then there arises the question: what should be the equation of motion in higher dimensions? Note that we are here not seeking an effective equation that takes into account some semi-classical corrections; instead we are asking for a classical equation in higher dimensions. To this aim, the first and foremost requirement is that it should be of second order, which uniquely picks out the Lovelock polynomial action in which each term comes with a dimensionful coupling constant. Also note that the Lovelock Lagrangian is the most general invariant that can be constructed from the Riemann tensor, giving the second order equation of motion.

On the other hand one can carry on with the Einstein equation itself, which is valid in all dimensions $d \geq 3$. This would, however, not be the most general equation in dimensions $>4$, while the Lovelock polynomial action would give the most general equation for all $d \geq 2 N+1$, and it includes GR for $N=1$. The problem with the Lovelock equation is that it has a dimensionful coupling for each $N$, and there is no way to determine more than one coupling by measuring the strength of the field, which is the only one. Thus there is arbitrariness in fixing the couplings.

There is one way out: that by invoking some property of gravity if we can justify that the Lovelock polynomial should involve only one $N$ th order term. That would then be what we have called the pure Lovelock case. That property is indeed a universalization of the kinematic property; i.e. that gravity be kinematic (non-existence of non-trivial vacuum spacetime) in all odd, $d=2 N+1$, dimensions. Thus the kinematic property uniquely picks out pure Lovelock gravity. This equation would be valid only for two (odd and even, $d=2 N+1,2 N+2$ ) dimensions because otherwise the kinematic property would be violated.

Note that the pure Lovelock equation [6] has several interesting and desirable features. For instance even though the equation is completely free from the Einstein term yet a static vacuum solution with $\Lambda$ asymptotically goes over to an Einstein-dS solution in the given dimension [11]. This is quite remarkable: that the pure Lovelock solution includes Einstein gravity asymptotically even though the equation is completely free from it. Similarly, bound orbits around a static black hole exist in pure Lovelock gravity in all even, $d=2 N+2$, dimensions, in contrast for Einstein gravity they do exist only in 4 dimension and not otherwise [12]. Note that the existence of bound orbits is required for having stable structures. If gravity has to have a similar behavior to the Einstein gravity in 3 and 4 dimensions in higher dimensions, stable structures should exist. Also thermodynamical parameters, temperature and entropy, obey a universal relation with the horizon radius in all odd and even, $d=2 N+1,2 N+2$, dimensions. In particular, the entropy always goes as the square of the horizon radius in all even dimensions [13].

Thus the pure Lovelock equation has all the features that one could have asked for a gravitational equation. The newly recognized kinematic property is clearly its distinguishing feature. It is thus the right gravitational equation [6] in higher dimensions, $d=2 N+1,2 N+2$. That is, for each $N$, the equation is only for the corresponding two odd and even dimensions; for instance for $N=1$, the Einstein equation is only for $d=3,4$, for $N=2$, the pure GB equation only for $d=5,6$, and so on. The Einstein equation is therefore good only for 3 and 4 dimensions, and in higher dimensions we should go over to the next order of $N$. Thus pure Lovelock gravity is a new paradigm for higher dimensions, and it is the kinematic property that has played the key distinguishing role.

\section{Discussion}

By appealing to a universalization of the kinematic property (that there exists no non-trivial vacuum solution in odd dimensions) for all odd dimensions, we have arrived at the unique gravitational equation which is pure Lovelock involving only one $N$ th order term. Thus the kinematic property plays the key role as a determining criterion as well as a guiding principle for gravitational dynamics in higher dimensions. We have just universalized the already existing property in Einstein gravity to get to the proper equation in higher dimensions. A good and enlightening generalization of a theory always stems from extending some key property beyond the normal premise of the theory, and then the existing theory gets automatically included in the new theory. Pure Lovelock gravity, which uniquely incorporates the kinematic property for all odd, $d=2 N+1$, dimensions, includes Einstein gravity for $N=1$.

The principal aim of the essay is to demonstrate the key distinguishing role the kinematic property plays in picking up the right equation for gravity in higher dimensions. Having done that let us ask: what more does it entail? 
There is a famous BTZ black hole solution [14] in 3 dimensions which is a $\Lambda$-vacuum solution. Note that it is the presence of $\Lambda$ that makes spacetime non-flat. For Einstein gravity, it can therefore occur only in 3 dimensions and not otherwise. Since pure Lovelock gravity is kinematic in all odd dimensions, analogs of BTZ black holes would exist in all odd, $d=2 N+1$, dimensions [2]

All this is very fine, however, the key question remains: how does the higher dimensional equation influence the 4spacetime we live in? The braneworld model [15] that envisages propagation of gravity in higher dimensions but not deep enough predicts a $1 / r^{3}$ correction to the Newtonian potential on the brane corresponding to an AdS bulk. The situation should be similar in what we are proposing, except perhaps we would rather employ a pure Gauss-Bonnet equation in the bulk rather than Einstein. This may not, however, be very relevant in so far as the AdS (which is a solution of the pure GB equation as well) bulk is concerned. The situation would be different if we consider a pure GB-BTZ black hole so that Weyl curvature in the 5 dimensional bulk is non-zero, which will project down on the brane as tracefree black radiation in the equation. Then we would have a black hole on the brane given by the Reissner-Nordstrom metric obtained by Dadhich et al. [16] where $Q^{2}$ is not the Maxwell charge but it is the Weyl charge appearing in the metric as $-Q^{2} / r^{2}$. It is yet very insightful, however, there exists no complete solution of the bulk-brane system.

It is indeed very remarkable and insightful that a universalization of a certain gravitational property uniquely picks out an equation in higher dimensions. In the same vein, let us further ask: is there any other similar instance of insightful deduction? The one thing that comes to mind is the question: how should vacuum energy gravitate $[1,17]$ ? It was argued that vacuum energy was on the same footing as gravitational field energy. Both are created by matter and hence have no independent existence on their own, and therefore they should not gravitate through a stress tensor in the equation independent of whether a stress tensor could be written or not. Clearly we write no stress tensor for a gravitational field energy on the right, and in fact it gravitates in a much subtler manner. It gravitates by enlarging the spacetime framework, by curving 3-space [18]. That is why Newton's inverse square law remains intact in GR. Something similar should happen for vacuum energy.

It is therefore a matter of principle that vacuum energy should not gravitate through a stress tensor but instead by enlargement of the framework. This we would not know unless we have a quantum theory of gravity. In any case, $\Lambda$ becomes free from the Planck length and hence it could, as a true constant of spacetime structure [1], have any value as being determined by acceleration of the Universe $[19,20]$. Thus it gets liberated and has nothing to do with vacuum energy $[1,17]$. At a conceptual level this is a very important realization.

The discovery of GR was solely driven by principles and concepts, and hence in its centennial year the present exercise is a fitting tribute to that spirit of doing science and to its great creator.

Acknowledgments The author warmly thanks the Albert Einstein Institute, Golm, where the manuscript was written, for a summer visit.

Open Access This article is distributed under the terms of the Creative Commons Attribution 4.0 International License (http://creativecomm ons.org/licenses/by/4.0/), which permits unrestricted use, distribution, and reproduction in any medium, provided you give appropriate credit to the original author(s) and the source, provide a link to the Creative Commons license, and indicate if changes were made.

Funded by $\mathrm{SCOAP}^{3}$.

\section{References}

1. N. Dadhich, Int. J. Mod. Phys. D 20, 2739 (2011). arXiv:1105.3396

2. N. Dadhich, S.G. Ghosh, S. Jhingan, Phy. Lett. B 711, 196 (2012). arXiv: 1202.4575

3. X.O. Camanho, N. Dadhich, Eur. Phys. J. C (2016). doi:10.1140/ epjc/s10052-016-3891-5

4. N. Dadhich, Pramana 74, 875 (2010). arXiv:0802.3034

5. D. Kastor, Class. Quantum Gravity 29, 155007 (2012). arXiv: 1202.5287

6. N. Dadhich, The gravitational equation in higher dimensions, in Relativity and Gravitation: 100 Years After Einstein in Prague, ed. by J. Bicak, T. Ledvinka (Springer, Berlin, 2013). arXiv: 1210.3022

7. N. Dadhich, A. Molina, X.O. Camanho, Class. Quantum Gravity 32, 175016 (2015)

8. N. Dadhich, Universalization as a physical guiding principle. arXiv:gr-qc/0311028

9. N. Dadhich, Universal features of gravity and higher dimensions. arXiv: 1105.0988

10. N. Dadhich, Universality, gravity, the enigmatic $\Lambda$ and beyond, in Proceedings of 12th Regional Conference on Mathematical Physics, ed. by M.J. Islam, F. Hussain, A. Qadir, H. Saleem (World Scientific, Singapore, 2006). arXiv:hep-th/0509126

11. N. Dadhich, J.M. Pons, K. Prabhu, Gen. Relativ. Gravit. 45, 1131 (2013). arXiv:1201.4994

12. N. Dadhich, S.G. Ghosh, S. Jhingan, Phys. Rev. D 88, 124040 (2013). arXiv:1308.4770

13. N. Dadhich, J.M. Pons, K. Prabhu, Gen. Relativ. Gravit. 44, 2595 (2012). arXiv:1110.0673

14. M. Banados, C. Teitelboim, J. Zanelli, Phys. Rev. Lett. 69, 1849 (1992)

15. L. Randall, R. Sundrum, Phys. Rev. Lett. 83, 4690 (1999)

16. N. Dadhich, R. Maartens, P. Papadopoulos, V. Rezania, Phys. Lett. B 487, 1 (2000)

17. N. Dadhich, $\Lambda$ is a constant of spacetime structure and has nothing to do with vacuum energy, in The Talk Given in the Workshop on Questioning Fundamental Physical Principles, CERN, 6-9 May 2014

18. N. Dadhich, Curr. Sci. 109, 260 (2015). arXiv:1206.0635

19. S. Perlmutter et al., Astrophys. J. 483, 566 (1997)

20. S. Perlmutter et al., Nature 391, 51 (1998) 